\title{
Cardiac MRI in this Era
}

\author{
Mona Bhatia ${ }^{1}$ Parveen Kumar ${ }^{1}$ \\ ${ }^{1}$ Department of Imaging, Fortis Escort Heart Institute, New Delhi,
India
}

Ind J Car Dis Wom:2020;5:335-342

\begin{abstract}
Address for correspondence Mona Bhatia, MD, FRCR(UK), FSCCT(USA), FSCMR (USA), Department of Imaging, Fortis Escort Heart Institute, Okhla Road, New Delhi 110025, India (e-mail: monabhatia1@gmail.com).
\end{abstract} 西

\author{
Abstract \\ Keywords \\ - cardiac mass \\ - cardiac MRI \\ - cardiomyopathy \\ - CMR \\ - ischemic \\ cardiomyopathy
}

Cardiac MRI (CMR) in this era is fast emerging as an invaluable tool in assessment of a large gamut of cardiac pathologies to not only ascertain the diagnosis but also assess severity, enable prognostication of disease process, guide management, and follow-up patients. CMR is today the gold standard for accurate and reproducible cardiac functional assessment, with excellent soft-tissue contrast, and ability to evaluate myocardial involvement even without contrast objectively via mapping techniques. CMR is thus fast emerging as the modality of choice and exponentially growing for cardiac assessment.

\section{Introduction}

Globally, cardiovascular morbidities and mortalities account for a large percentage of patients. Similar statistics are present in India. Early diagnosis with accurate evaluation is critical; hence, imaging plays a lead role. Various modalities exist with a gamut of radiography, echocardiography, CT, MRI, nuclear scintigraphy, and invasive angiography. MRI has emerged as a winner and leads the array of diagnostic modalities in this era. The chief advantages of MRI include noninvasiveness, lack of ionizing radiations, and excellent temporal and spatial resolution. ${ }^{1,2}$ Recent advances in technology allow wide range of applications in myocardial viability, cardiomyopathies, congenital and valvular heart diseases, pericardial pathologies, cardiac masses, and aortic and pulmonary vessels evaluation. Emerging applications of MRI include assessment of myocardial perfusion, ischemia, coronary artery and vessel wall analysis, atherosclerotic plaque imaging, myocardial tissue characterization (T1, T2 mapping), and even therapeutic MRI prior to electrophysiology studies and interventions. Therefore, CMR shows great promise in cardiac and vascular evaluation in current times.

\section{Technical Aspects of Cardiac MRI}

Different sequences have been designed, aiming at different imaging requirements. These include black blood and white blood images. ECG gating is prerequisite for optimum image quality. The black blood images are particularly useful to evaluate the cardiac anatomy using time of flight and echo time variations to generate spin echo (SE), fast spin echo (FSE), and single shot turbo fast spin echo sequences. The white blood images are useful to demonstrate cardiac motion and valvular diseases. Bright blood sequences include gradient-echo (GRE) and refocused steady-state free precession (SSFP) sequences.

Other bright blood imaging techniques include flow-dependent time-of-flight and MR angiography, which may be three-dimensional (3D) in nature postgadolinium injection. Time of flight imaging is based on phenomena of flow-related enhancement, which is used to visualize flow within the vessels without giving contrast. 3D MR angiography postgadolinium injection is useful for vascular evaluation of pulmonary vessels, aortography, and coronary bypass grafts.
DOI https://doi.org/

10.1055/s-0040-1722383.
(C) 2020. Women in Cardiology and Related Sciences.

This is an open access article published by Thieme under the terms of the Creative Commons Attribution-NonDerivative-NonCommercial-License, permitting copying and reproduction so long as the original work is given appropriate credit. Contents may not be used for commercial purposes, or adapted, remixed, transformed or built upon. (https://creativecommons.org/licenses/by-nc-nd/4.0/).

Thieme Medical and Scientific Publishers Pvt. Ltd. A-12, 2nd Floor, Sector 2, Noida-201301 UP, India 
First-pass postcontrast MRI is used to assess the microcirculation of the myocardium. Late gadolinium enhancement MRI (LGE-MRI) is used for myocardial tissue characterization, scar/fibrosis detection and quantification, and distinguishing ischemic cardiomyopathy from nonischemic cardiomyopathy. The commonly used contrast agent is water-soluble gadolinium. Myocardial tagging is used to evaluate myocardial function. The dark grid aids evaluation of the myocardial motion through the entire cardiac cycle with accurate evaluation of displacement, deformation and myocardial strain, thus enabling evaluation of regional myocardial function. Myocardial T1 mapping is used to assess the degree of myocardial fibrosis invisible to LGE-MRI. T2 mapping is used to quantify myocardial edema. T2* mapping is used to quantify the degree of iron deposition in the myocardium, especially in patients with hemosiderosis, thalassemia major and repeated blood transfusion. ${ }^{3}$

The reasons for the emergence and dependence on CMR is the high soft-tissue contrast, lack of ionizing radiations, large field of view, accuracy, versatility, functional assessment, reproducibility ability of myocardial tissue characterization, and suitability for treatment monitoring and follow-up of disease progression . The limitations include high cost, availability, dyspneic and claustrophobic patients, patients with MR conditional and unsafe devices, and need for cardiac and respiratory gating. Contrast reactions like nephrogenic systemic fibrosis pose furthermore restrictions. The important challenges in cardiac imaging include respiratory and cardiac motion. While breath holding may eliminate the former, the latter requires electrocardiography (ECG) gating to ensure imaging in the desired phase as systolic, diastolic or through the entire cardiac cycle. Cine imaging with ECG triggered segmentation permits evaluation of the cardiac and valvular function besides blood flow, myocardial contractility and wall motion.

\section{Imaging Planes}

There are three standard cardiac planes including short axis (SA) (-Fig. 1), horizontal long axis (four-chamber view) (-Fig. 2), and vertical long axis (two-chamber view) (-Fig. 3). These planes are defined on axial body plane images along a straight line, extending from center of mitral valve to cardiac apex (long axis of heart). While the SA plane is at right angle to the long axis, the vertical long axis plane is orthogonal to the SA plane. The horizontal long axis is prescribed along a horizontal plane at right angle to the SA plane. Other commonly used planes include the three-chamber view, in the form of the left ventricular outflow tract (LVOT) view and right ventricular outflow tract (RVOT) view, and the oblique sagittal view and "three points" plane view by connecting three points along a selected coronary artery. ${ }^{4}$

\section{Indications and Applications}

\section{Ischemic Heart Disease}

Coronary artery disease is the most often the cause of ischemic heart disease. Ischemic heart disease manifests as

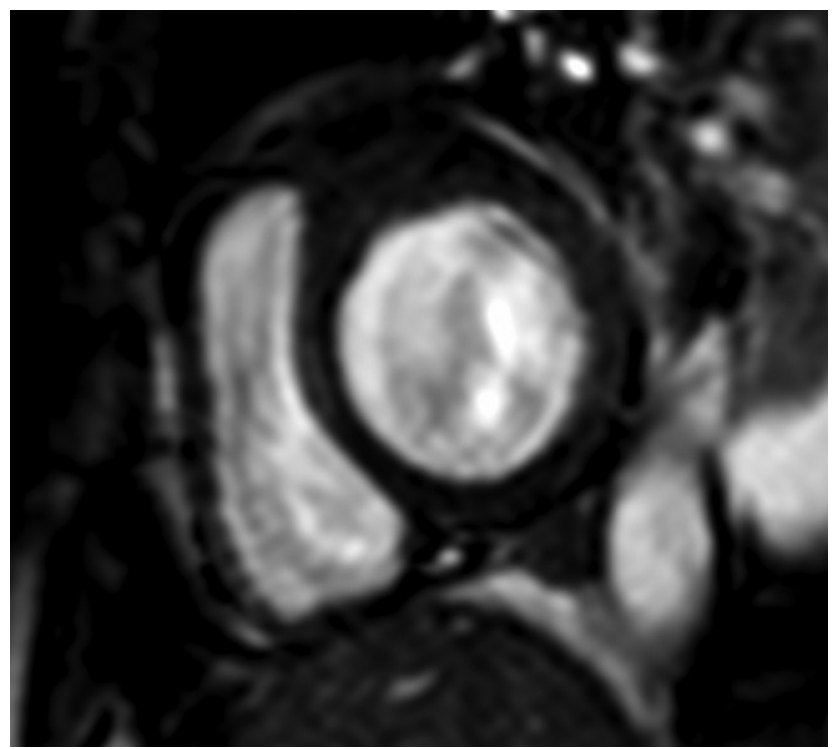

Fig. 1 Short-axis, BTFE CMR image displaying the white blood image of the left and right ventricles. Abbreviation: BTFE, balanced turbo field echo.

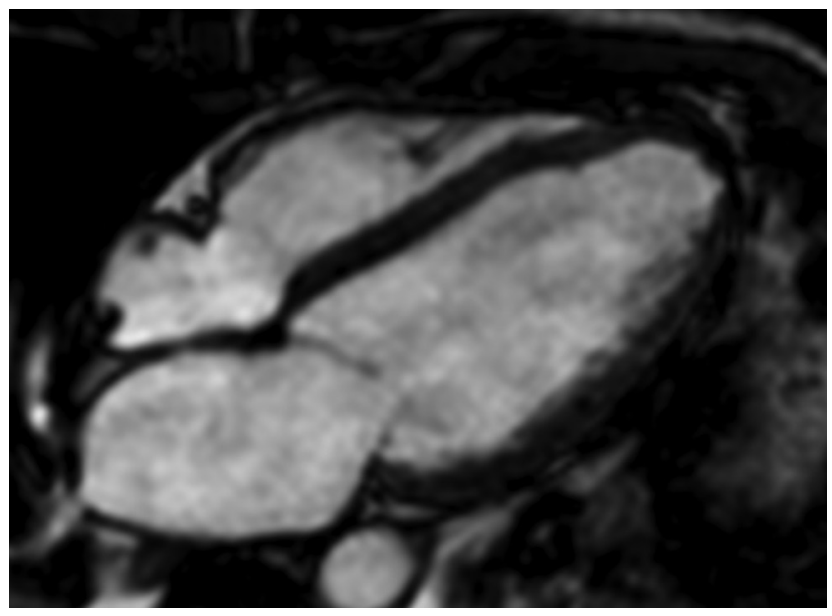

Fig. 2 Four-chamber view, BTFE CMR image displaying all four chambers and atrioventricular valves. Abbreviation: BTFE, balanced turbo field echo.

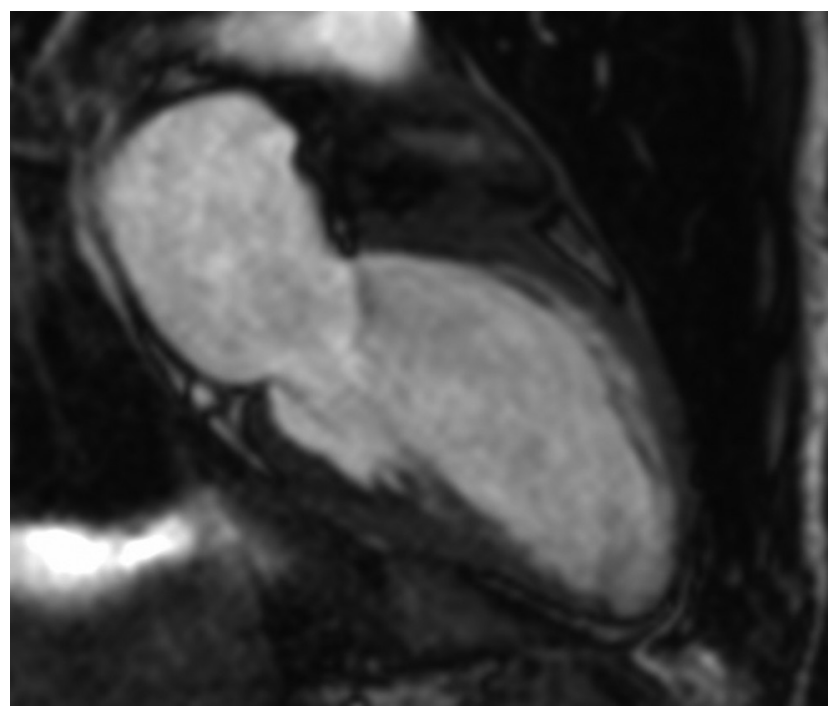

Fig. 3 Two-chamber view, BTFE CMR image displaying the left atrium, left ventricle and intervening mitral valve. Abbreviation: BTFE, balanced turbo field echo. 
ventricular dysfunction, resulting from coronary artery disease or myocardial infarction. CMR enables excellent evaluation of not only the cardiac structures but also the gold standard for cardiac function. A comprehensive protocol provides an accurate quantification of cardiac function, wall edema, perfusion defects on first-pass perfusion, and viability in single setting. Dynamic cine MR sequences such as SSFP provide a means of reliable evaluation of chamber volumes and function. Ventricular function can be assessed quantitatively (absolute or relative wall thickening and wall motion) or qualitatively (normal, akinetic, dyskinetic or hypokinetic). Myocardial tagging is a noninvasive technique to calculate myocardial strains. It creates grid lines on myocardium which allows analysis of displacement and strain on the myocardium through different phases of the cardiac cycle. Myocardial perfusion is assessed by monitoring the passage of contrast bolus through the chambers and the myocardium using the ultrafast cine MR sequences. Perfusion imaging can be done in resting state or after administration of adenosine (stress MR). Homogenous enhancement of the myocardium implies normal perfusion, while presence of dark areas during first-pass perfusion implies areas of perfusion defect and typically respect the territories of coronary arteries. ${ }^{5}$

Late gadolinium hyperenhancement (LGE) is associated with myocyte necrosis; hence, implies infarction reliably (-Fig. 4). Postgadolinium evaluation of first-pass perfusion and enhancement pattern, following adequate delay, are useful to assess viability, which is an important determinant of functional recovery. The presence and quantification of perfusion defect, together with imaging for delayed enhancement, predicts scar and fibrosis. ${ }^{6}$ Regional wall motion abnormalities are seen in myocardial ischemia and

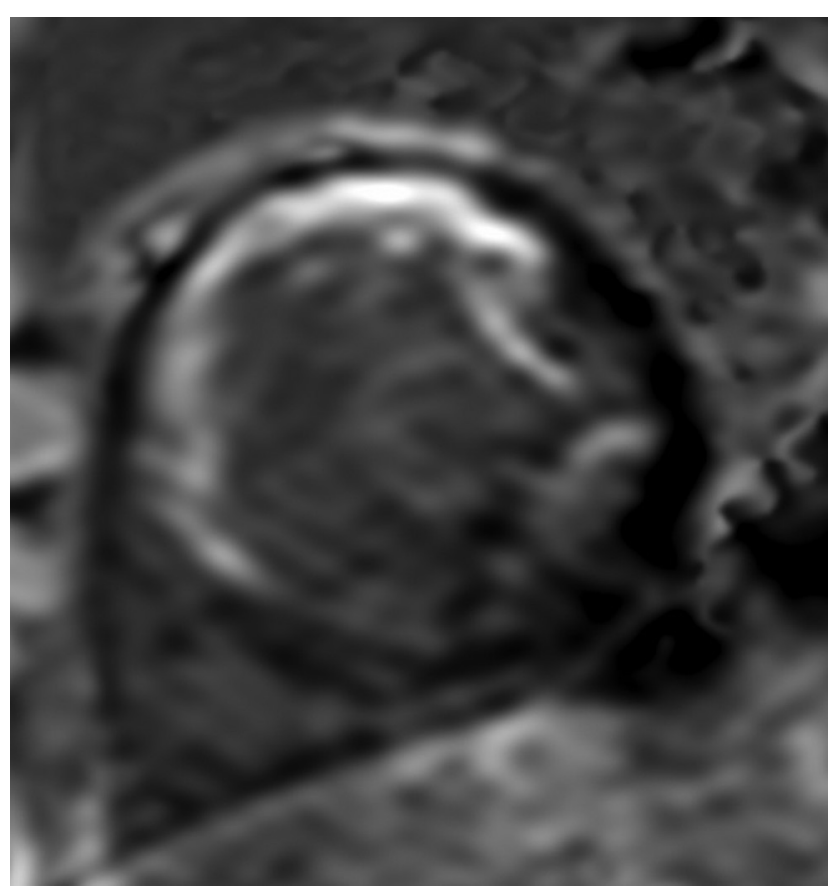

Fig. 4 Short-axis, LGE CMR image in midventricular level demonstrating wall thinning and transmural enhancement along the LAD territory in a case of myocardial infarction. Abbreviation: LGE, late gadolinium enhancement. infarction. One method to differentiate acute infarction from chronic infarction is the presence of increased signal indicative of edema on T2-weighted imaging. Microvascular obstruction (MVO) may be seen as a dark area surrounded by enhancement when the infarct is severe. MVO implies poor prognosis and may therefore impact management strategy. It is important to keep in mind that the differential diagnosis of delayed enhancement may vary and include not only acute and chronic infarcts but also various inflammatory, infectious and infiltrative pathologies. The pattern of enhancement may vary from midwall, epicardial or global extending from subendocardial to transmural in nature.

The usefulness of LGE MRI is multifold on account of its high-spatial resolution, together with the fact that cine imaging enables evaluation of wall motion abnormalities in addition to cardiac morphology. MRI is extremely accurate in subendocardial infarction assessment, and on comparison with its competition, LGE MRI has excellent correlation with dobutamine stress echocardiography and positron emission tomography (PET). A sensitivity of $96 \%$ and specificity of $84 \%$ has been demonstrated in a study comparing LGE MRI and 18-fluorodeoxyglucose PET as the standard. ${ }^{7}$ MRI is superior to single-photon emission computed tomography (SPECT), ${ }^{6}$ as studies demonstrate that $47 \%$ of subendocardial infarctions may be missed on SPECT.

CMR is also valuable in the assessment of postinfarction complications. The complications include aneurysmal change with or without intracavitary thrombi, pericarditis, and associated pericardial effusion. MRI can differentiate true aneurysm from pseudoaneurysm. Ventricular thrombi, which are easily missed on echocardiography, are best visualized on early contrast-enhanced sequences as intracavitary filling defects. Similarly, MI-associated pericarditis is depicted on late CEMRI as enhancing pericardium.

Recent technological innovations like T1 and T2 mapping allow evaluation of MR images. Native T1 mapping can differentiate infarcted myocardium from microvascular obstruction. T2 mapping can identify myocardial edema. Postcontrast T1 mapping can quantify the infarcted myocardium. ${ }^{8}$

\section{Hypertrophic Cardiomyopathy}

Cardiac MRI is extremely useful in evaluation of echo-poor segments of the myocardium to assess the extent of hypertrophic cardiomyopathy ( $\mathrm{HCM}$ ), especially in areas poorly visualized on echocardiography as the ventricular apex. Different morphological forms of HCM include asymmetric, concentric, septal, or papillary muscle hypertrophy. Asymmetric septal hypertrophy is a frequently discussed form and may result in LVOT obstruction, referred to as hypertrophic obstructive cardiomyopathy. The diagnostic criteria of asymmetric or septal HCM includes greater than or equal to $15 \mathrm{~mm}$ septal thickness or the ratio of the septal to inferior wall thickness is greater than 1.5 at the midventricular level ( - Fig. 5). CMR can differentiate obstructive from nonobstructive types of HCM, based on gradient measurement between LVOT and aorta at rest and/or with provocation. Furthermore, it can demonstrate systolic anterior motion of mitral leaflets. LGE helps to evaluate fibrosis and 


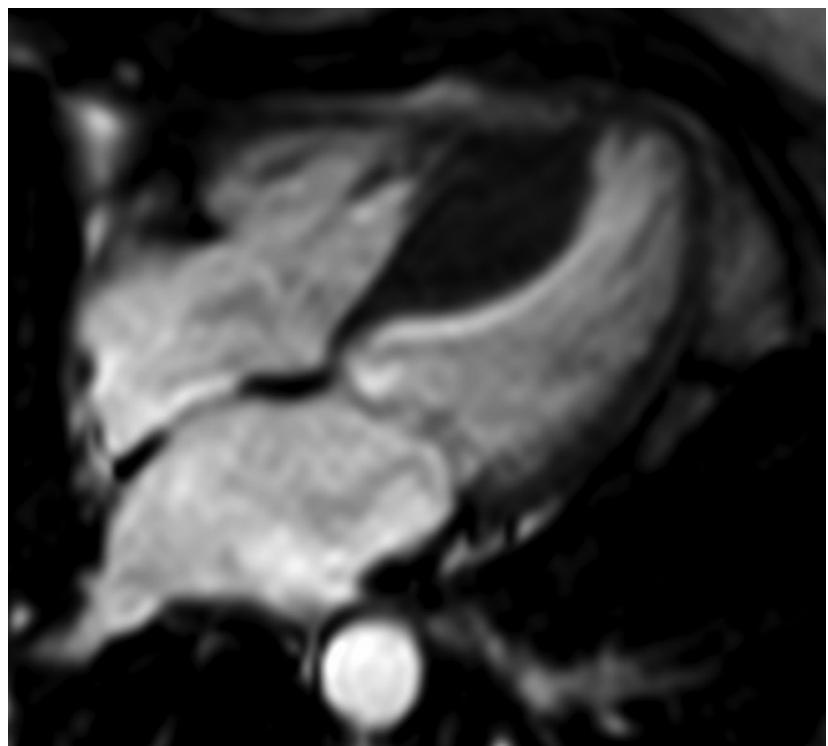

Fig. 5 Four-chamber, BTFE CMR image depicting midcavity asymmetric septal hypertrophy, consistent with hypertrophy cardiomyopathy. Abbreviation: BTFE, balanced turbo field echo.

scarring in hypertrophied myocardium, which may act as potential sites or nidus for arrythmias. CMR for follow-up of patients is useful after septal ablation or septal resection. Native T1 mapping is also increasingly been utilized for evaluation of myocardial fibrosis, which is undetected by LGE. Native and postcontrast $\mathrm{T} 1$ values correlate with disease severity. ${ }^{9-12}$

\section{Restrictive Cardiomyopathy}

The condition is a consequence of increased myocardial stiffness with impaired ventricular filling. Thus, the left ventricular size and systolic function are normal, while diastolic dysfunction may be severe, and biatrial enlargement may be seen. MRI can evaluate restrictive diseases as:

\section{Sarcoidosis}

CMR is gold standard for assessing the chamber size and ejection fraction. The signal intensity of sarcoid lesions varies with evolving stages of the disease, ranging from T2-weighted high-signal intensity to a high-signal intensity ring and a central low-intensity on T1/T2-weighted imaging. The principle method of detecting cardiac sarcoidosis relies on LGE which shows midwall and epicardial pattern of enhancements ( - Fig. 6). Advanced stages show myocardial wall or septal thinning, left ventricular systolic and diastolic dysfunction, and aneurysm formation. ${ }^{13-15}$

\section{Amyloidosis}

Ventricular wall thickening may raise the suspicion of amyloidosis with associated atrial septal thickening. The atrial septal thickness of $6 \mathrm{~mm}$ is found to be fairly specific for amyloid infiltration. The pattern of LGE in amyloidosis can be focal or global ( - Fig. 7) and be associated with worse prognosis in amyloidosis. Furthermore, survival is poor in transmural enhancement versus subendocardial type.

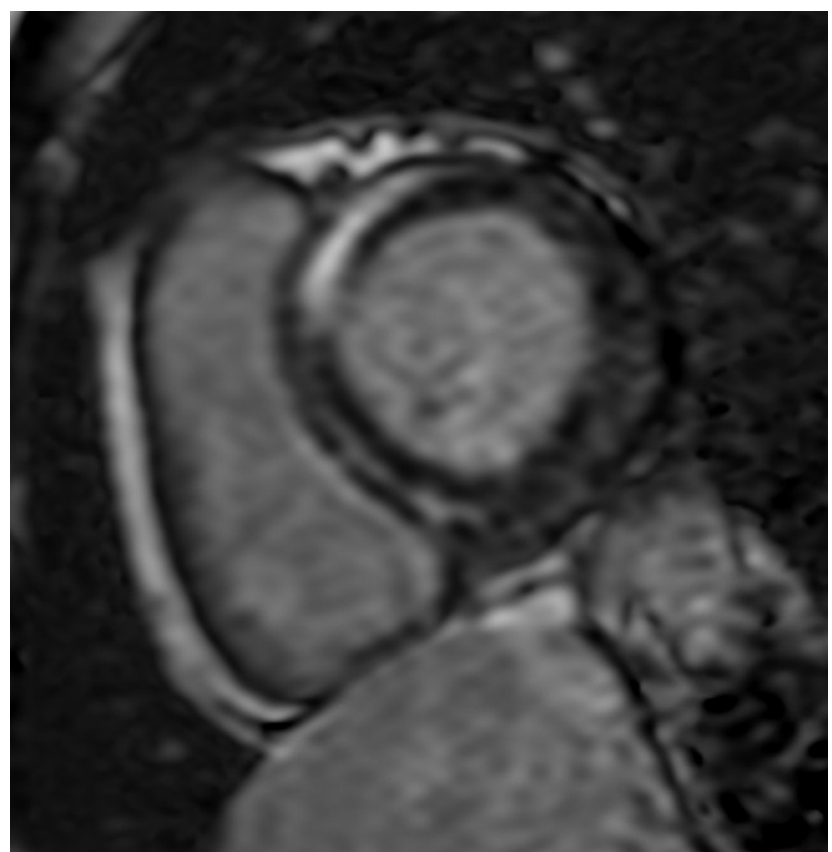

Fig. 6 Short-axis, LGE PSIR CMR image demonstrating linear enhancement along the midwall aspect of the septum in a case of sarcoidosis. Abbreviations: LGE, late gadolinium enhancement, PSIR, phase-sensitive inversion recovery.

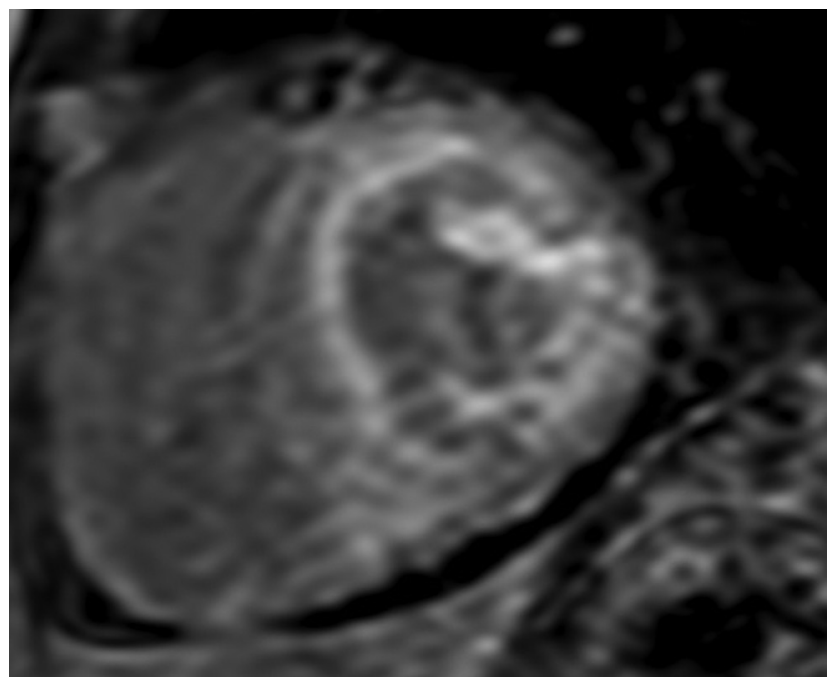

Fig. 7 Short-axis, LGE CMR image at midventricular level demonstrating global subendocardial LGE with dark blood pool, consistent with amyloidosis. Abbreviation: LGE, late gadolinium enhancement.

T1 mapping is another useful tool. Cardiac amyloidosis is associated with marked increase in native T1 and ECV values. ${ }^{16,17}$

\section{Endomyocardial Fibrosis (EMF)}

Also termed Loeffler endocarditis, it demonstrates subendocardial fibrosis, which may be extensive with associated apical thrombus and diastolic dysfunction. LGE is an important determinant of tissue characterization. The typically described pattern is "double $V$ sign," which comprises the three-layered pattern of central and peripheral myocardium, 
enhancing endomyocardium and overlying thrombus (-Fig. 8). MRI is excellent not only in morphology but also functional quantification. ${ }^{18,19}$

\section{Hemochromatosis}

T1- and T2-weighted mapping sequences of the dysfunctional myocardium demonstrate signal loss in native studies, especially when there is an associated signal loss in the liver, confirming iron deposition and systemic hemochromatosis.

\section{Dilated Cardiomyopathy (DCM)}

A dilated left ventricle is an important indication of DCM with decreased systolic function, while the coronary arteries do not demonstrate significant disease or chronic pressure overload. Causes are related to intrinsic myocardial damage. CMR can analyze wall thickness, impaired fiber shortening, end-systolic wall stress, dilated heart chambers, and delayed-enhancement characteristics. LGE images are critical to differentiate ischemic from nonischemic dilated cardiomyopathy. The midwall fibrosis is an independent predictor of morbidity and mortality after cardiac resynchronization therapy. ${ }^{20-22}$

\section{Myocarditis}

Myocarditis is one of the most frequent indications for CMR. It is an important diagnostic tool to rule out myocarditis in patients presenting with normal coronaries, or in patients with atypical symptoms. CMR targets several key features of myocarditis including wall edema, necrosis, scar, and accompanying pericardial effusion. CMR is an extremely useful tool to delineate inflamed myocardium prior to planning endomyocardial biopsy. Myocardial mapping techniques such as native T1 and T2 mapping may show values as a result of inflammation and help in objective quantification of myocardial edema. ${ }^{23,24}$

\section{Pericardial Disease}

Besides pericardial morphology, CMR provides comprehensive evaluation of the impact of pericardial disease on

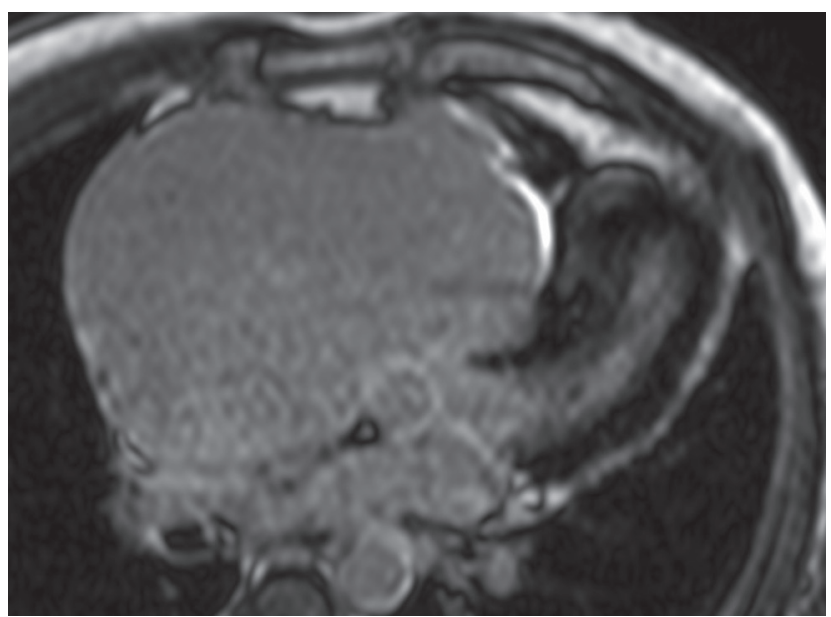

Fig. 8 Four-chamber, PSIR image demonstrating obliteration of the RV apex with fibrosis in a case of endomyocardial fibrosis. Abbreviation: PSIR, phase-sensitive inversion recovery. cardiac structure and function. While the normal pericardium appears as a thin hypointense structure $(2 \mathrm{~mm})$ with epicardial and pericardial fat on either side on T1-weighted spin-echo imaging, thickened pericardium in excess of $2 \mathrm{~mm}$ is suggestive of inflammation and may be associated with pericardial effusion. A pericardium thickness greater than $4 \mathrm{~mm}$ is abnormal and may imply fibrous pericarditis ${ }^{1,2}$ ( - Fig. 9). Pericardial effusion is often associated with complications such as septations, adhesions and/or loculations, which differentiate transudative and exudative from hemorrhagic fluid based on CMR characteristics and postcontrast enhancement. While simple effusions are hypointense on T1 and hyperintense on T2-weighted images, chylous and hemorrhagic effusions appear hyperintense on T1-weighted imaging. CMR also provides information on functional consequences of rigid and thickened pericardium. Tagging sequences causes distortion of the tag lines in cases where there are pericardial adhesions. Evaluation of subtle changes in pericardium are not always possible on echocardiography. Velocity-encoded CMR is helpful to demonstrate pericarditis associated restrictive filling, typically seen as increased early and decreased late filling. ${ }^{25}$

\section{Arrhythmogenic Right Ventricular Dysplasia}

Arrhythmogenic right ventricular cardiomyopathy (ARVC) is an inherited cardiomyopathy, and its arrhythmogenic nature may result in sudden cardiac death in the young. The most frequently involved sites are basal right ventricle with sparing of RV apex. MRI is currently the modality of choice with revised "task force" criteria. The CMR features of ARVC include segmental and/or global dilatation of the RV, with akinetic and dyskinetic bulges, focal or regional wall motion abnormalities, trabeculations and aneurysmal outpouching. Postcontrast delayed enhancement plays a crucial role in

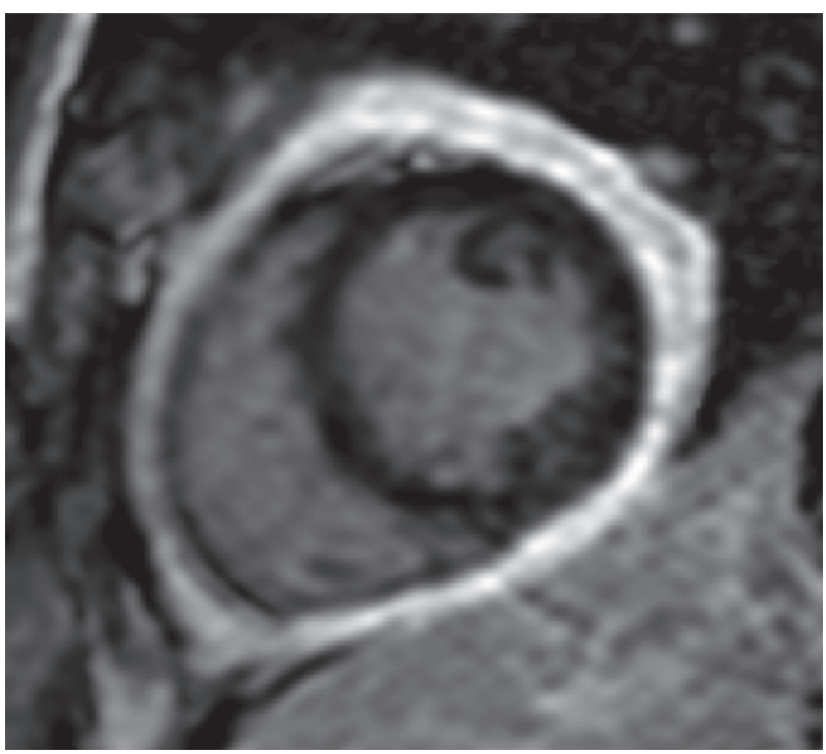

Fig. 9 Short-axis, LGE CMR image at basal ventricular level demonstrating thickened enhancing pericardium, consistent with constrictive pericarditis. Abbreviation: LGE, late gadolinium enhancement. 
evaluating the presence of fibrofatty involvement and myocardial fibrosis. ${ }^{26}$ ( - Fig. 10)

The diagnosis of ARVC is often challenging, particularly since echocardiography has limited capability in RV evaluation; hence, imaging today relies on the modified ARVC task force criteria. These criteria involve multiple modalities such as echocardiography, CMR, angiography, ECG and family history. The findings are divided into major and minor criteria. The diagnosis of ARVC is established, based on presence of either of two major criteria, one major and two minor criteria, or four minor criteria. The CMR findings which are indicative and compatible with major criteria include regional RV akinesia or dyskinesia or dyssynchronous contraction of RV and one of the following: Ratio of RV end-diastolic volume index $($ RVEDV/BSA) $\geq 110 \mathrm{~mL} / \mathrm{m} 2$ (male) or $\geq 100 \mathrm{~mL} / \mathrm{m} 2$ (female) or RV ejection fraction $\leq 40 \%$. The CMR findings indicative and compatible with minor criteria include regional akinesia or dyskinesia or dyssynchronous contraction of RV and one of the following: Ratio of RVEDV/BSA $\geq 100$ to $110 \mathrm{~mL} / \mathrm{m} 2$ (male) or $\geq 90$ to $100 \mathrm{~mL} / \mathrm{m} 2$ (female) or RV ejection fraction $>40$ to $\leq 45 \%{ }^{26}$

\section{Cardiac Masses}

CMR provides comprehensive evaluation of cardiac tumors. It can assess the primary mass (size, location, signal characteristics, and borders), locoregional relations of tumor, tumor vascularity, and tumor-related complications (valve stenosis, valve insufficiency, or pericardial effusion). CMR can characterize the tissue components within tumors including fat, fibrosis, hemorrhage, calcification, and cystic changes. ${ }^{27}$

CMR is the modality of choice to differentiate tumor from thrombi (-Fig. 11). A thrombus unlike a tumor may

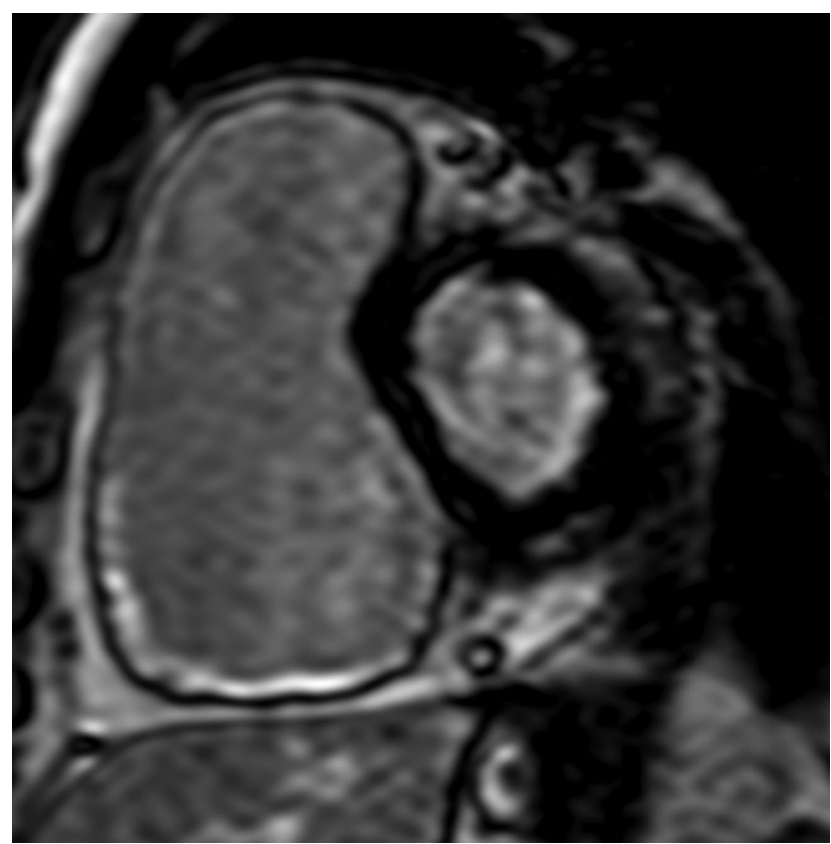

Fig. 10 Short-axis, LGE PSIR CMR image demonstrating RV dilatation with fibrosis along the free wall of RV in a case of arrythmogenic right ventricular cardiomyopathy. Abbreviations: LGE, late gadolinium enhancement, PSIR, phase-sensitive inversion recovery. have a low-signal intensity on GRE images on account of deoxyhemoglobin. Myxoma arises from the interatrial septum, being pedunculated or sessile, with smooth contours. A thrombus is often located in the atrial appendage with broad-based and irregular contours. Myxoma can prolapse through the mitral valve at cine imaging. In the event of a diagnostic dilemma, where a myxoma appears hypointense secondary to calcification or hemorrhage, the differentiation is easily done postcontrast, as enhancement occurs in the tumor but not in the thrombus. Occasionally, the normal cardiac structure may simulate a cardiac mass. Cine MR can also assist differentiate between two entities based on contractions through the cardiac cycle. MRI is also useful in the evaluation of other cardiac tumors such as metastasis (-Fig. 12), fibroma, hemangioma, lymphoma, pheochromocytoma, angiosarcoma and malignant fibrous histiocytoma. The malignant tumors are more likely to be necrotic, inhomogeneous, and heterogeneously enhancing with associated edema and invasion of surrounding structures. ${ }^{28,29}$

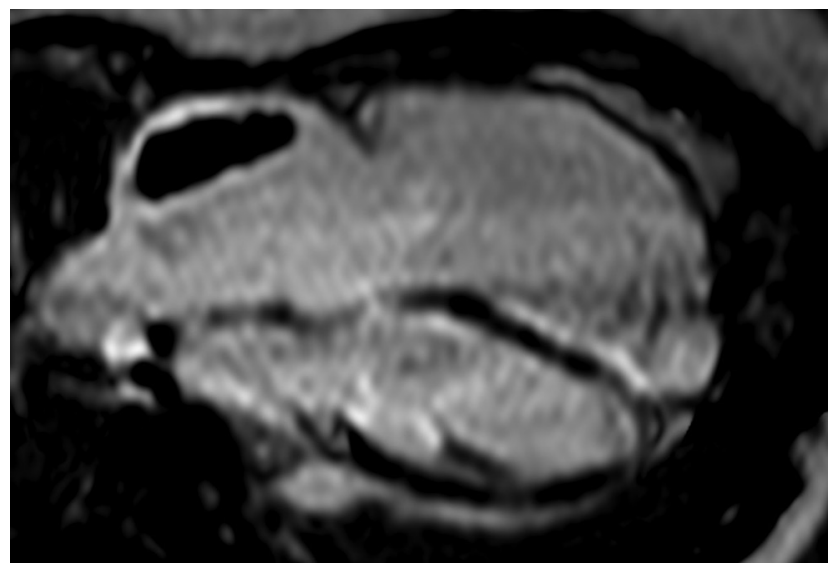

Fig. 11 Four-chamber, LGE PSIR CMR image demonstrating a nonenhancing right atrial intracavitory thrombus. Abbreviations: LGE, late gadolinium enhancement, PSIR, phase-sensitive inversion recovery.

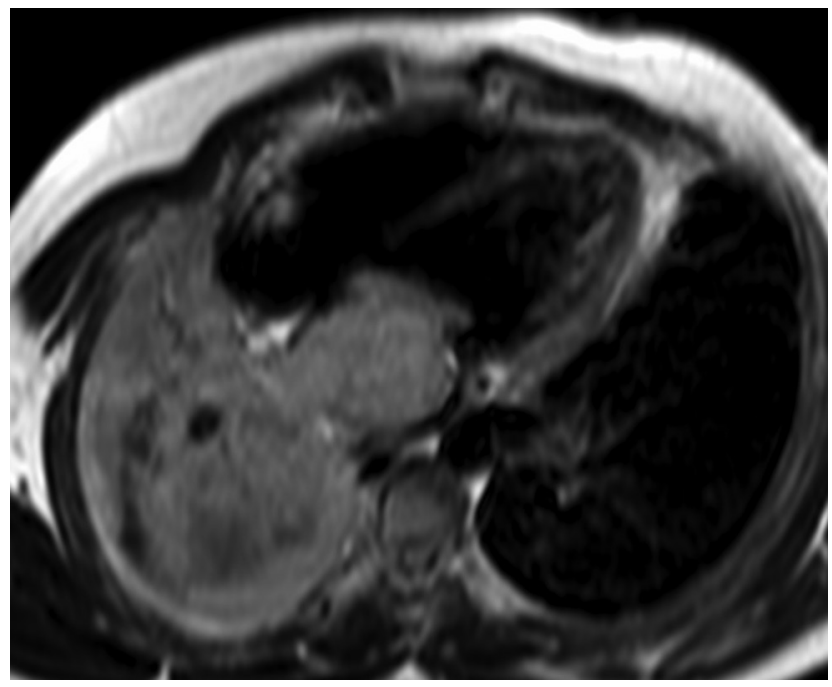

Fig. 12 Four-chamber CMR image depicting a large intra and extracardiac mass. 


\section{Valvular Disease}

CMR allows full visualization of valves, direct measurement of valve area, their inflow and outflow tracts, and associated great vessel anatomy. Signal void extent and length of time is indicative of severity of aortic stenosis; similarly, the signal loss area is indicative of severity of mitral regurgitation. ${ }^{30}$ The sensitivity (98\%), specificity (95\%), and accuracy (97\%) of MRI to diagnose and evaluate aortic and mitral regurgitation is extremely high. ${ }^{31}$ The severity of valvular stenosis can be assessed by phase contrast techniques based on valve orifice area and transvalvular pressure gradients. Aortic and mitral valves areas measure 2.5 to $3.5 \mathrm{~cm}^{2}$ and 4 to $6 \mathrm{~cm}^{2}$, respectively; severe stenosis is indicated with an area less than $0.8 \mathrm{~cm}^{2}$ and $1 \mathrm{~cm}^{2}$, respectively. ${ }^{6}$ Furthermore, the ventricular volumetrics and myocardial mass are indicators of impact of valvular disease on ventricular functions.

\section{Coronary Magnetic Resonance Angiography}

CMR allows noninvasive and ionizing radiation-free detection of lumen narrowing coronary artery disease. Its ability to quantify the flow is another added advantage. The common challenges include small caliber of coronaries, constant respiratory and cardiac motion, and surrounding bright epicardial fat on MRI. Recent advances in CMR sequences, 3D techniques and postcontrast coronary MRA will make it possible to assess beyond the origin and proximal segments of coronaries and bypass graft patency, although CT has superior accuracy. Sternal wires, metallic clips, and various graft markers' artifacts may limit the evaluation. ${ }^{32,33}$

\section{Future Perspectives}

CMR methodology is evolving at a rapid pace. The latest mapping techniques of $\mathrm{T} 1$ and $\mathrm{T} 2$ sequences ensures objective evaluation of T1, T2, and ECV of the myocardium ( - Fig. 13).

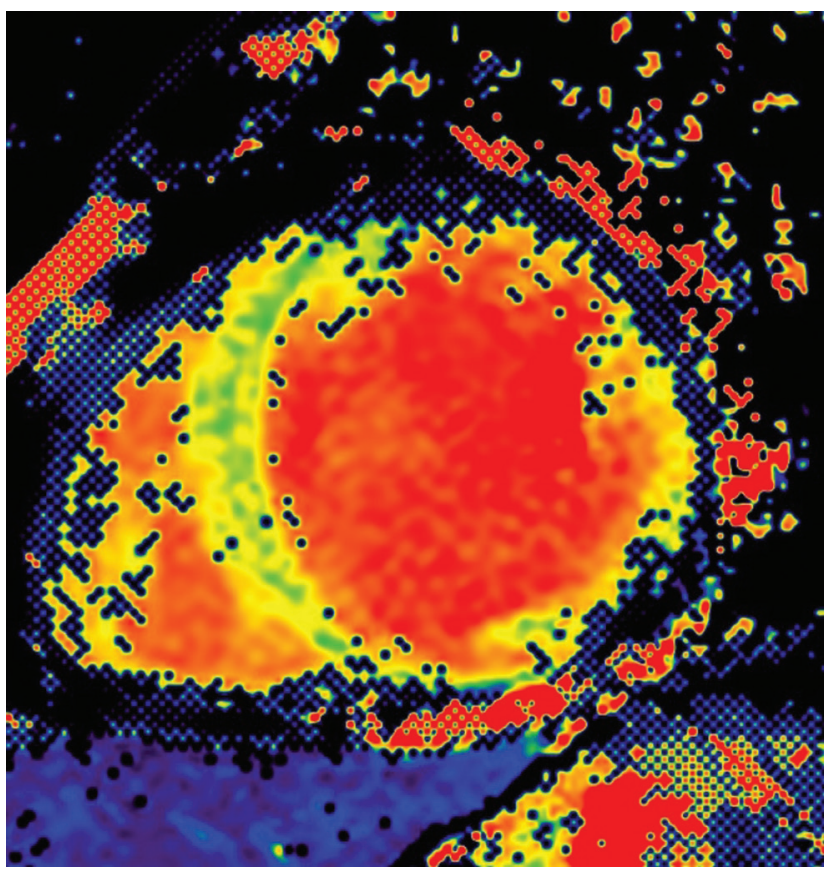

Fig. 13 Short axis CMR image for T1 mapping.
Molecular imaging and interventional MRI techniques are now evolving to create stem cell therapies. Iron-based, contrast-labeled stem cells enable localization postimplantation for follow-up of stem cell therapy in the heart. ${ }^{34-36}$ Ironloaded precursor cells in scarred myocardium can also be evaluated by MRI. ${ }^{37}$ Furthermore, there is ongoing research for the use of machine learning relevant to CMR in the areas of image analysis, acquisition and reconstruction, diagnostic evaluation, and prognostication. Machine learning has reduced the time required for segmentation and analysis significantly.

\section{Conclusion}

CMR has fast evolved into an exceptional modality for cardiovascular disease assessment. Its major advantages are superior soft-tissue contrast, nonionizing nature, and high-spatial and temporal resolution, enabling high quality assessment of the cardiac structure and function; most importantly, the myocardial characterization, which is being correlated with histology. CMR is poised to change the manner in which cardiology diagnostics, management, and follow-up will be performed.

\section{Indian Perspective}

Cardiovascular diseases have for a long time been a leading cause of morbidity in India; however, the turn of the century is now showing epidemiological move toward increasing risk factors and prevalence of cardiovascular diseases, with the same having emerged as the leading cause of mortality in India, figures rising to $25 \%$. Given that CVD is resulting in a critical economic and social impact on India, surveillance, early diagnosis, and appropriate management are becoming increasingly important for effective impact. Despite the huge benefits, there are multiple limitations related to the scope of cardiac MRI in India, from availability and access to equipment, cost, trained personnel, and physician awareness. Cardiac MRI capable centers pan India are extremely few. India is however standing at the brink of change, ushering in technological breakthroughs in medical science and research. The national cardiology and imaging societies are coming forward in a concerted approach to spread the awareness and education about the evolving role of CMR in cardiovascular disease. A revolution is definitely brewing and the healthcare industry promises to be a front runner of this revolution. Most certainly, "this is the era of Cardiac MRI" in cardiology practice.

\section{Conflict of Interest}

None.

\section{References}

1 Lima JA, Desai MY. Cardiovascular magnetic resonance imaging: current and emerging applications. J Am Coll Cardiol 2004;44(6):1164-1171

2 Edelman RR. Contrast-enhanced MR imaging of the heart: overview of the literature. Radiology 2004;232(3):653-668 
3 Azevedo C, Cury R, Cardiovascular MR. Imaging: current status and future directions. Magn Reson Imaging Clin N Am 2019;27(3):xvii-xviii

4 Ginat DT, Fong MW, Tuttle DJ, Hobbs SK, Vyas RC. Cardiac imaging: part 1, MR pulse sequences, imaging planes, and basic anatomy. AJR Am J Roentgenol 2011;197(4):808-815

5 Reeder SB, Du YP, Lima JA, Bluemke DA. Advanced cardiac MR imaging of ischemic heart disease. Radiographics 2001; 21(4):1047-1074

6 Lin EC. Cardiac MRI, technical aspects primer. Available at: http://emedicine.medscape.com/article/352250-overview. Accessed May 2014

7 Kühl HP, Beek AM, van der Weerdt AP, et al. Myocardial viability in chronic ischemic heart disease: comparison of contrast-enhanced magnetic resonance imaging with (18) F-fluorodeoxyglucose positron emission tomography. J Am Coll Cardiol 2003;41(8):1341-1348

8 Kim PK, Hong YJ, Im DJ, et al. Myocardial T1 and T2 Mapping: Techniques and Clinical Applications. Korean J Radiol 2017;18(1):113-131

9 Friedrich MG. Magnetic resonance imaging in cardiomyopathies. J Cardiovasc Magn Reson 2000;2(1):67-82

10 Luckie M, Khattar RS. Systolic anterior motion of the mitral valve-beyond hypertrophic cardiomyopathy. Heart 2008; 94(11):1383-1385

11 Halpern EJ, Cardiac morphology and function. In: Clinical cardiac CT: Anatomy and Function. New York, NY: Thieme; 2008 166-175

12 Shah JS, Esteban MT, Thaman R, et al. Prevalence of exercise-induced left ventricular outflow tract obstruction in symptomatic patients with non-obstructive hypertrophic cardiomyopathy. Heart 2008;94(10):1288-1294

13 Martusewicz-Boros MM, Boros PW, Wiatr E, Kempisty A, Piotrowska-Kownacka D, Roszkowski-Śliż K. Cardiac sarcoidosis: is it more common in men? Hai 2016;194(1):61-66

14 Terasaki F, Yoshinaga K. New guidelines for diagnosis of cardiac sarcoidosis in Japan. Ann Nucl Cardiol 2017;3(1):42-45

15 Birnie DH, Sauer $\mathrm{WH}$, Bogun $\mathrm{F}$, et al. HRS expert consensus statement on the diagnosis and management of arrhythmias associated with cardiac sarcoidosis. Heart Rhythm 2014;11(7):1305-1323

16 Fontana M, Pica S, Reant P, et al. Prognostic value of late gadolinium enhancement cardiovascular magnetic resonance in cardiac amyloidosis. Circulation 2015;132(16):1570-1579

17 Muchtar E, Blauwet LA, Gertz MA. Restrictive cardiomyopathy: genetics, pathogenesis, clinical manifestations, diagnosis, and therapy. Circ Res 2017;121(7):819-837

18 de Carvalho FP, Azevedo CF. Comprehensive assessment of endomyocardial fibrosis with cardiac mri: morphology, function, and tissue characterization. Radiographics 2020; 40(2):336-353

19 Beaton A, Mocumbi AO. Diagnosis and management of endomyocardial fibrosis. Cardiol Clin 2017;35(1):87-98

20 Hare JM, The dilated, restrictive and infiltrative cardiomyopathies. In: Bonow RO, Mana DL, Zipes DP, et al, eds. Braunwald's
Heart Disease: A Text Book Of Cardiovascular Medicine. 9th ed. Philadilphia (PA): Saunders Elsevier; 2011

21 To AC, Desai MY. Role of cardiac magnetic resonance imaging in assessing ischemic and nonischemic cardiomyopathies. Expert Rev Cardiovasc Ther 2012;10(2):223-233

22 Tigen K, Karaahmet T, Kirma C, et al. Diffuse late gadolinium enhancement by cardiovascular magnetic resonance predicts significant intraventricular systolic dyssynchrony in patients with non-ischemic dilated cardiomyopathy. J Am Soc Echocardiogr 2010;23(4):416-422

23 CooperLTJr.Myocarditis.NEnglJMed2009;360(15):1526-1538

24 Kindermann I, Barth C, Mahfoud F, et al. Update on myocarditis. J Am Coll Cardiol 2012;59(9):779-792

25 Bogaert J, Francone M. Cardiovascular magnetic resonance in pericardial diseases. J Cardiovasc Magn Reson 2009;11(1):14

26 te Riele AS, Tandri H, Bluemke DA. Arrhythmogenic right ventricular cardiomyopathy (ARVC): cardiovascular magnetic resonance update. J Cardiovasc Magn Reson 2014;16(1):50

27 Li X, Chen Y, Liu J, et al. Cardiac magnetic resonance imaging of primary cardiac tumors. Quant Imaging Med Surg 2020;10(1):294-313

28 Motwani M, Kidambi A, Herzog BA, Uddin A, Greenwood JP, Plein S. MR imaging of cardiac tumors and masses: a review of methods and clinical applications. Radiology 2013; 268(1):26-43

29 Tumma R, Dong W, Wang J, Litt H, Han Y. Evaluation of cardiac masses by CMR-strengths and pitfalls: a tertiary center experience. Int J Cardiovasc Imaging 2016;32(6):913-920

30 Myerson SG. Heart valve disease: investigation by cardiovascular magnetic resonance. J Cardiovasc Magn Reson 2012;14(1):7

31 Pohost GM, Hung L, Doyle M. Clinical use of cardiovascular magnetic resonance. Circulation 2003;108(6):647-653

32 Achenbach S, Ropers D, Regenfus M, et al. Noninvasive coronary angiography by magnetic resonance imaging, electron-beam computed tomography, and multislice computed tomography. Am J Cardiol 2001;88(2A) :70E-73E

33 Ropers D, Regenfus M, Stilianakis N, et al. A direct comparison of noninvasive coronary angiography by electron beam tomography and navigator-echo-based magnetic resonance imaging for the detection of restenosis following coronary angioplasty. Invest Radiol 2002;37(7):386-392

34 Orlic D, Hill JM, Arai AE. Stem cells for myocardial regeneration. Circ Res 2002;91(12):1092-1102

35 Hill JM, Dick AJ, Raman VK, et al. Serial cardiac magnetic resonance imaging of injected mesenchymal stem cells. Circulation 2003;108(8):1009-1014

36 Kraitchman DL, Heldman AW, Atalar E, et al. In vivo magnetic resonance imaging of mesenchymal stem cells in myocardial infarction. Circulation 2003;107(18):2290-2293

37 Garot J, Unterseeh T, Teiger E, et al. Magnetic resonance imaging of targeted catheter-based implantation of myogenic precursor cells into infarcted left ventricular myocardium. J Am Coll Cardiol 2003;41(10):1841-1846 